\title{
Model-free, Model-based, and General Intelligence
}

\author{
Hector Geffner ${ }^{1,2}$ \\ ${ }^{1}$ Universitat Pompeu Fabra, Roc Boronat 138, 08032 Barcelona, Spain \\ 2 ICREA, Pg. Lluís Companys 23, 08010 Barcelona, Spain \\ hector.geffner@upf.edu
}

\begin{abstract}
During the $60 \mathrm{~s}$ and $70 \mathrm{~s}$, AI researchers explored intuitions about intelligence by writing programs that displayed intelligent behavior. Many good ideas came out from this work but programs written by hand were not robust or general. After the 80s, research increasingly shifted to the development of learners capable of inferring behavior and functions from experience and data, and solvers capable of tackling well-defined but intractable models like SAT, classical planning, Bayesian networks, and POMDPs. The learning approach has achieved considerable success but results in black boxes that do not have the flexibility, transparency, and generality of their model-based counterparts. Modelbased approaches, on the other hand, require models and scalable algorithms. Model-free learners and model-based solvers have close parallels with Systems 1 and 2 in current theories of the human mind: the first, a fast, opaque, and inflexible intuitive mind; the second, a slow, transparent, and flexible analytical mind. In this paper, I review developments in AI and draw on these theories to discuss the gap between model-free learners and model-based solvers, a gap that needs to be bridged in order to have intelligent systems that are robust and general.
\end{abstract}

\section{Introduction}

The current excitement about $\mathrm{AI}$ is the result of a number of breakthroughs in machine learning. Some of these developments are true AI milestones, like the programs that achieve top world performance in games such as Chess and Go by learning from self-play only [Silver et al., 2017a; 2017b]. These are no small accomplishments and most of them have to do with deep learning [LeCun et al., 2015] and deep reinforcement learning [Mnih et al., 2015]. The goal of this paper is to place these developments in perspective, in particular by comparing model-free learners with modelbased solvers. Solvers are programs that accept instances of different types of models, like SAT, classical planning, Bayesian networks, and POMDPs, and automatically compute their solutions. Learners and solvers have interesting similarities and differences. Both are concerned with the derivation of functions $f$ for mapping inputs $x$ into outputs, but while learners derive the function $f$ from data or experience, solvers compute the output $f(x)$ for each given input $x$ from a model. Interestingly the scope and characteristics of model-free learners and model-based solvers have close parallels with those of the two systems or processes that are currently assumed to make up the human mind [Kahneman, 2011; Evans and Stanovich, 2013]: one, a fast, opaque, and inflexible intuitive mind (System 1); the other, a slow, transparent, and general analytical mind (System 2). A number of works have analyzed the limitations of deep learning, often placing the emphasis on the need to construct and use models [Lake et al., 2017; Darwiche, 2017; Pearl, 2018; Marcus, 2018b]. In this paper, I place the emphasis on solvers, and in particular planners, which require both models and general, scalable algorithms. The Systems 1 and 2 perspective will be useful for understanding the scope of learners and solvers, and the need for a tight integration.

The paper is organized as follows. I trace some history, look at learners, solvers, and planners, which are a particular type of solvers, and address the similarities and differences between learners and solvers, and the challenge of integrating them.

\section{Programming, AI, and AI Programming}

Artificial Intelligence is a brain child of Alan Turing and his universal computer [Turing, 1936]. Turing was not only a logician and the father of the modern computer but also the first modern programmer. Programming played a key role in the early days of AI and in the selection of papers for the seminal Computers and Thought book where each chapter described "a working computer program" [Feigenbaum and Feldman, 1963]. The emphasis on working programs, however, was not shared by everyone. John McCarthy, who was both the father of the most popular AI programming language at the time (Lisp) and the person that gave AI its name, was putting the basis for the logical approach to AI where the emphasis was not in the use of representations but in their expressiveness and semantics [McCarthy, 1958]. Yet such dissenting views were not common and are not to be found in the CT book. Several of the key AI contributions in 60s, $70 \mathrm{~s}$, and $80 \mathrm{~s}$ had to do indeed with programming and the representation of knowledge in programs, and this includes 
Lisp and functional programming, Prolog and logic programming, rule-based programming, expert systems shells, frames, scripts, and semantic networks [Charniak et al., 1980; Norvig, 1992].

\section{The Problem of Generality}

Many great ideas came out of this work but there was a problem: the programs were not sufficiently robust or general, and they tended to fail on examples or scenarios not anticipated by the programmer. It was natural to put the blame on the knowledge that the programs were missing but it was not clear then how to proceed. Some programmers narrowed down the domain and scope of the programs so that all the relevant knowledge could be made explicit. This was the 'expert systems' approach. Some took the programs as illustrations or demonstrations of potential capabilities which were not actually delivered. Some decided to sit down and write down all the relevant commonsense knowledge. This was the motivation underlying projects like CYC [Lenat et al., 1990]. McCarthy discussed related issues in his 1971 ACM Turing lecture entitled "Generality in Artificial Intelligence", published in revised form many years later [McCarthy, 1987].

The limitations of AI programs for exhibiting general intelligence not tied to toy worlds or narrow domains led to an impasse in the 80s, one of whose effects was a methodological shift in which research increasingly moved away from writing programs for ill-defined problems to designing algorithms for well-defined mathematical tasks. The algorithms are general in the sense that they are not tied to particular examples but to certain classes of models and tasks expressed in mathematical form. The new programs, learners and solvers, have a crisp functionality and both can be seen as computing functions that map inputs into outputs (Figure 1).

\section{Learners}

The current excitement about AI in academia and in industry is the result of a number of breakthroughs that have pushed the state-of-the-art in tasks such as image understanding, speech recognition, and challenging games [Krizhevsky et al., 2012; Graves et al., 2013; Mnih et al., 2015], in certain cases delivering superhuman performance while starting with basic knowledge of the rules of the game only [Silver $e t$ $a l ., 2017 \mathrm{~b}]$. These developments have to do with two classes of learners: deep learners and deep reinforcement learners. While these are not the only approaches pursued in machine learning, they are the ones behind these milestones and the ones on which I focus.

In both deep learning (DL) and deep reinforcement learning (DRL), training results in a function $f_{\theta}$ that has a fixed structure, given by a deep neural network [LeCun et al., 2015], and a number of adjustable parameters $\theta$. In DL, the input vector $x$ may represent an image and the output $f_{\theta}(x)$, a classification label, a probability distribution over the possible labels, or object labels with suitable bounding boxes. In DRL, the input $x$ may represent the state of a dynamic system or game, and the output $f_{\theta}(x)$, the value of the state, the action to be done in the state, or a probability distribution over such actions. The key difference between DL and DRL is in

$$
\text { Input } x \Longrightarrow \text { FUNCTION } f \Longrightarrow \text { Output } f(x)
$$

Figure 1: Learners and solvers map inputs into outputs. Learners derive the function $f$ from data or experience. Solvers derive the value of the function $f(x)$ for each given input $x$ from a model.

the way in which the functions $f_{\theta}$ are learned during training. Deep learning is a supervised method where the parameters $\theta$ are learned by minimizing an error function that depends on the inputs and target outputs in a training set. Deep reinforcement learning, on the other hand, is a non-supervised method that learns from experience, where the error function depends on the value of states and their successors. In both DL and DRL, the most common algorithm for minimizing the error function is stochastic gradient descent where the parameter vector $\theta$ is modified incrementally by taking steps in the direction of the gradient. Similar optimization algorithms are used in policy-based DRL where the function $f_{\theta}$ represents a policy.

The basic ideas underlying DL and DRL methods are not new and can be traced back to the neural network and reinforcement learning algorithms of the 80s and 90s [Rumelhart and McClelland, 1986; LeCun et al., 1989; Sutton and Barto, 1998]. The recent successes have to do with the gains in computational power and the ability to use deeper nets on more data. The use of these methods on problems that have attracted and spurred commercial interest has helped as well. A common question that arises is what are the limits of these methods. One important restriction is that the inputs $x$ and outputs y of neural nets have a bounded, fixed size. This limitation is not relevant for learning to play chess or Go whose boards have a fixed size, but is relevant for tackling, for example, arbitrary instances of the much simpler Blocks world. Attention mechanisms have been proposed as a way to deal with arbitrarily large inputs [Graves et al., 2014], but such mechanisms introduce partial observability, which is another challenge in learning. Indeed, as one moves away from the basic setting of DL and DRL, the methods, the results, and the evaluation standards, all look somehow weaker.

\section{Solvers}

The second type of programs that we consider are solvers. Solvers take a convenient description of a particular model instance (a classical planning problem, a constraint satisfaction problem, and so on) and automatically compute its solution. Solvers can also be thought as computing a function $f$ mapping inputs $x$ into outputs, the difference is that they work out of the box without training by computing the output $f(x)$ lazily for each given input $x$. The target $f(x)$ is given implicitly by the model. For a SAT solver, the inputs $x$ are formulas in conjunctive normal form, and the output $f(x)$ tells whether the formula $x$ is satisfiable or not. For a classical planner, the inputs are classical planning problems $x$ and the output is a plan that solves the problem. Solvers have been developed for a variety of models that include constraint satisfaction problems (CSPs), SAT, Bayesian networks, classical planning, fully and partially observable non-deterministic and 
stochastic planning problems (FONDPs, PONDPs, MDPs, and POMDPs), general game playing, and answer set programming among others [Dechter, 2003; Biere et al., 2009; Pearl, 1988; Geffner and Bonet, 2013; Bertsekas, 1995; Kaelbling et al., 1998; Genesereth et al., 2005; Gebser et al., 2012]. Solvers are general as they must deal with any problem that fits the model: any classical planning problem, any CSP, etc. This presents a crisp computational challenge, as all models are computationally intractable, with complete algorithms running in time that is exponential in the number of problem variables or worse. The challenge is to push this exponential explosion as far as possible, as solvers should not break down on a problem merely because it has many variables. To achieve this, domain-independent solvers must be able to exploit the structure of the given problems so that their performance over a given domain can approach the performance of a domain-specific solver. The computational value of solving techniques is assessed experimentally and in most cases by means of competitions. Competitions have helped to generate hundreds of problems used as benchmarks, have set standards for the encodings of problems, and have facilitated the empirical evaluation of algorithms. The focus on models and solvers that can scale up has acted as a powerful filter on ideas and techniques, setting up a clear distinction between the ideas that look well from those that actually work well. We illustrate some of these ideas in the context of planning to make the comparison between learners and solvers more concrete.

\section{Planners}

Planners are solvers for models that involve goal-directed behavior. Planners and planning models come in many forms depending on a number of dimensions including: 1) uncertainty about the initial situation and action transitions, 2) type of sensing, 3) representation of uncertainty, and 4) objectives. The simplest type of planning, classical planning, involves no uncertainty about the initial situation or action effects, hence no uncertainty or sensing, and the objective is to reach some condition. MDPs have stochastic actions and full state observability and the objectives are described in two different ways: as goals to be reached with probability 1 by applying actions with positive costs (goal MDPs), or as discounted rewards to be collected (discounted reward MDPs). Other types of objectives considered in planning are temporally extended goals, like "visit rooms 5 and 7 forever", that are conditions on possibly infinite state trajectories [Camacho et al., 2017]. Three other relevant dimensions in planning are whether 5) models are expressed in compact or flat form, 6) solutions are sought off-line or on-line, and in the latter case, 7) which off-line solution form is sought. About the last point, partially observable problems can be solved optimally with policies that map belief states into actions but can also be approached effectively with simpler solution forms such as finite-state controllers. Many of these dimensions affect the complexity of planning. Focusing on the decision problems, classical planning is PSPACE-complete, while classical planning with a fixed horizon is NP-complete [Kautz and Selman, 1996]. Similarly, PONDPs expressed in compact form are
2-EXP complete [Rintanen, 2004], while computing memoryless policies for PONDPs expressed in flat form is NPcomplete [Chatterjee et al., 2016].

We look next at three powerful computational ideas in planning, relaxations, transformations, and width, drawing in each case contrasts between planners and learners.

\subsection{Relaxations}

The derivation of heuristics in classical planning for guiding the search for plans follows an old idea: if you do not know how to solve a problem, solve a simpler problem and use its solution as guidance for solving the original problem [Pearl, 1983]. In planning, the most useful simplification $P^{\prime}$ of a planning problem $P$ is the monotonic relaxation where the effects of the actions on the variables are made monotonic [McDermott, 1999; Bonet and Geffner, 2001]. For this, the states $\bar{s}$ of the relaxation $P^{\prime}$ are defined not as assignment of values $x$ to variables $X$ but as collection of atoms $X=x$. In the original problem $P$, if an action $a$ makes an atom $X=x^{\prime}$ true, it makes the atoms $X=x^{\prime}$ for $x \neq x^{\prime}$ false. In the monotonic relaxation, on the other hand, the new atom $X=x^{\prime}$ does not make the other atoms false. A result of this is that the monotonic relaxation $P^{\prime}$ becomes decomposable: a plan that achieves two atoms $X=x$ and $Y=y$ jointly in $P^{\prime}$ can be obtained by concatenating a plan for $X=x$ and a plan for $Y=y$. This idea can be used to compute plans for all atoms and hence for the conjunction of atoms in the goal of $P^{\prime}$ in low polynomial time. The heuristic $h(s)$ can be set then to the size of the plan in the relaxation obtained from the state $\bar{s}$ where the only true atoms are those which are true in $s$ [Hoffmann and Nebel, 2001]. ${ }^{1}$ Planners compute the heuristic value $h(s)$ for any state $s$ over any problem $P$ following variations of this procedure. This is why they are called domain-independent planners. Learners can infer the heuristic function $h$ over all the states $s$ of a single problem $P$ in a straightforward way, but they can't infer an heuristic function $h$ that is valid for all problems. This is natural: learners can deal with new problems only if they have acquired experience on related problems.

\subsection{Transformations}

Planners tend to work well even in large classical instances yet many problems do not have this form. Transformations are used to leverage on classical planners.

Goal recognition. Goal recognition is a classification problem where the hidden goal of an agent has to be uncovered from his observed behavior and a pool of possible goals. For example, an agent is observed to move up twice from the middle of the grid in Figure 2, and we want to determine to which of the marked targets he may be heading to. A goal recognition problem $P(\mathcal{G}, O)$ can be represented as a classical problem $P$ but with the goal replaced by a pool $\mathcal{G}$ of possible goals $G$ and a sequence of observed actions $O$. In the example, the reason that the observed agent is likely to be moving to one of

\footnotetext{
${ }^{1}$ When variables are boolean and actions are represented in STRIPS as precondition, add, and delete lists, the monotonic relaxation is known as the delete-relaxation, as the monotonic relaxation is obtained by making all delete lists empty.
} 

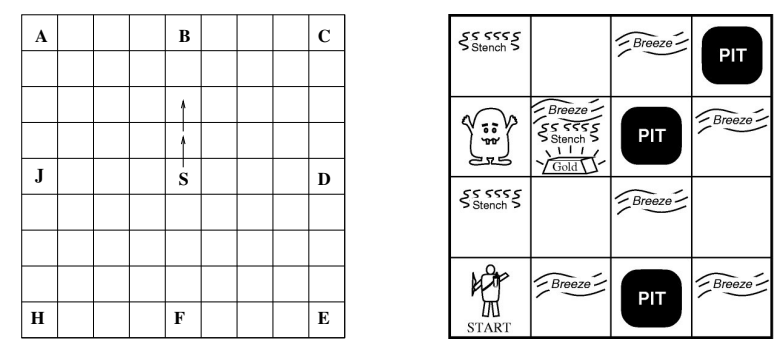

Figure 2: Left. Goal recognition: where is the agent heading to? Right. Partial observability: get the gold without knowing where the gold, pits, and wumpus are, using the sensors (see text).

targets in the top row is that it is not reasonable (cost-efficient) to reach the other targets by moving up. This can be formalized in terms of two cost measures (plan lengths) for each possible goal $G$ : the cost of reaching $G$ with a plan that complies with the observations, denoted $c^{+}(G)$, and the cost of reaching $G$ with a plan that does not comply with the observations, denoted $c^{-}(G)$. Bayes' rule says that the goal posterior is $P(G \mid O)=P(O \mid G) P(G) / P(O)$ where the priors $P(G)$ can be assumed to be given. The posterior $P(G \mid O)$ is then determined by the likelihoods $P(O \mid G)$ that express how well each of the possible goals $G$ predicts $O$. In [Ramírez and Geffner, 2010], the likelihood $P(O \mid G)$ is set to a monotonic function $f$ of the cost difference $\Delta(G)=c^{-}(G)-c^{+}(G)$ that expresses that the costlier that it is to reach $G$ with a plan that complies with $O$ in relation to one that does not, the less likely that it is the observation $O$ given $G$. The costs are computed by running a planner on problems obtained from the goal recognition problem $P(\mathcal{G}, O)$. The posterior distribution $P(G \mid O)$ for all goals $G \in \mathcal{G}$ is computed using Bayes' rule by calling a classical planner $2|\mathcal{G}|$ times. Once again, even if goal recognition is a classification problem, it is not possible to obtain a general, domain-independent account of goal recognition using learners instead of planners.

Partial observability. Figure 2 shows an instance of the Wumpus problem [Russell and Norvig, 2009] where an agent has to get the gold while avoiding the monster and the pits. The positions of the gold, the monster, and the pits, however, are not known to the agent that can sense stench if at distance one from the wumpus, a breeze, if at distance one from a pit, and a shiny object if in the same cell as the gold. The problem can be modeled as a POMDP or as a contingent planning problem but few planners scale up when the size of the grid is increased. Even if we focus on on-line planning methods, two challenges need to be addressed: tracking the beliefs about the true hidden state, and selecting the actions that take the agent to the goal or provide relevant information. The two challenges have been addressed jointly through a "planning under optimism" scheme where the partially observable problem $P$ is relaxed into a classical problem $K(P)$ inside a plan-execute-observe-and-replan loop [Bonet and Geffner, 2014]. The transformation $K(P)$ removes the uncertainty in $P$ in two steps. First, literals $L$ that represent that $L$ is true are replaced by two literals $K L$ and $K \neg L$, the first meaning $L$ is known to be true, the second meaning that $L$ is known to be false. Second, sensing the truth value of a positive literal $L$ that is currently unknown is mapped into two actions: one that predicts $K L$, and another that predicts $K \neg L$. The planexecute-observe-and-replan loop then executes the prefix of the plan obtained from $K(P)$ using a classical planner until a discrepancy is found between what has been predicted and what has been observed. In such a case, the observed literals are added, a new plan is computed, and the loop continues until the goal is reached. For problems with no dead ends and belief width 1 , the executions are guaranteed to reach the goal in a bounded number of steps [Bonet and Geffner, 2014]. Problems like Wumpus over 15x15 grids are solved consistently very fast. On the other hand, coverage over instances of problems like Minesweeper is not $100 \%$, which is natural as belief tracking in Minesweeper is NP-hard [Kaye, 2000] while incomplete and polynomial in the planner [Palacios and Geffner, 2009]. Learning approaches face two challenges in these types of problems: dealing with partial observability over instances of the same domain, and more critically, transferring useful knowledge from instances of one domain to instances of another.

Finite-state controllers and generalized planning. Finitestate controllers represent an action selection mechanism widely used in video-games and robotics. A finite-state controller for a partially observable problem $P$ with actions $a$ and observations $O$ can be characterized by a set of tuples $\left(q, o, a, q^{\prime}\right)$ with no pair of tuples sharing the first two components $q$ and $o$. Each such tuple prescribes the action $a$ to be done when the controller state is $q$ and the observation is $o$, switching then to the controller state $q^{\prime}$ (which may be equal to $q$ or not). A controller solves $P$ if starting in the distinguished controller state $q_{0}$, all the executions that are compatible with the controller reach a goal state. In the approach by Bonet et al. [2009], the problem $P$ is transformed into a classical planning problem $P^{\prime}$ whose actions are associated with each one of the possible tuples $\left(q, o, a, q^{\prime}\right)$, and where extra fluents $p_{q}$ and $p_{o}$ track the controller states and observations. The action $t=\left(q, o, a, q^{\prime}\right)$ behaves like the action $a$ but conditional on $p_{q}$ and $p_{o}$ being true, and setting $p_{q^{\prime}}$ to true. Figure 3 shows a problem where a visual-marker or eye (circle on the lower left) must be placed on top of a green block by moving it one cell at a time. The location of the green block is not known, and the observations are whether the cell currently marked contains a green block $(\mathrm{G})$, a non-green block (B), or neither (C), and whether this cell is at the level of the table $(\mathrm{T})$ or not $(-)$. The finite-state controller shown on the right has been computed by running a classical planner over the transformed problem. The controller moves the eye from left to right at the level of the table, and when it finds a block that is not green, it moves the eye up the tower and then down. Interestingly, this controller not only solves the problem shown on the left, but any modification of the problem resulting from changes in the number or configuration of blocks. This is an instance of what has been called oneshot learning: the controller obtained by the classical planner for one instance solves all instances of the problem. The task of computing solutions to families of planning problems, for example, all instances of Blocks world featuring blocks 

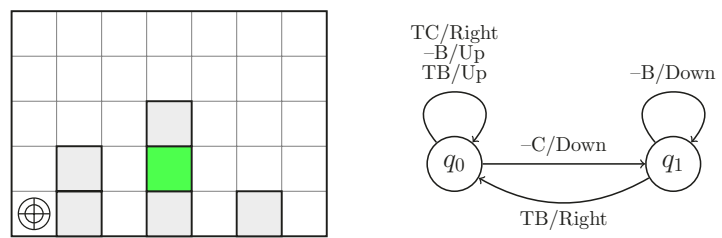

Figure 3: Left: Problem where a visual-marker (mark on the lower left cell) must be placed on top of a green block whose location is not known, by moving the mark one cell at a time, and by observing what's in the marked cell. Right: Two-state controller obtained with a classical planner. The controller solves the problem and any variation resulting from changes in the number or configuration of blocks. Edge $q \rightarrow q^{\prime}$ labeled $o / a$ represents tuple $\left(q, o, a, q^{\prime}\right)$.

$A$ and $B$ where the goal is $A$ on $B$, is called generalized planning [Srivastava et al., 2011; Hu and De Giacomo, 2011; Bonet et al., 2017]. Generalized planning provides an interesting bridge to work in DRL [Groshev et al., 2017] as a generalized plan is not just the output $f(x)$ for a particular planning instance $x$ (Figure 1), but the function $f$ itself. This is indeed a case where planners and learners aim at the same type of plans, ones using models, the others, experience.

\subsection{Width}

The third and last idea that we consider in planning is related to a very simple search algorithm called $\operatorname{IW}(k)$ that has some remarkable properties [Lipovetzky and Geffner, 2012]. The algorithm assumes that the states $s$ assign values to a number of boolean features $F$ that are given. In classical planning, the boolean features are the atoms of the problem but the algorithm applies to other settings as well. For $k=1$, IW(1) is a standard breadth-first search with just one change: if upon generation of a state $s$, there is no feature $f_{i}$ in $F$ such that $f_{i}$ is true in $s$ and false in all the states generated before $s$, the state $s$ is pruned. In other words, the only states that are not pruned are those that make some feature true for the first time. Such states are said to have novelty 1 . The number of states expanded by IW(1) is thus linear in $|F|$ and not exponential in $|F|$ as in breadth-first search. The algorithm $\operatorname{IW}(k)$ is IW(1) but applied to the larger feature set $F^{k}$ made up of the conjunctions of $k$ features from $F$. A basic property of $\operatorname{IW}(k)$ is that most classical planning benchmark domains can be formally shown to have a bounded and small width $w$ no greater than 2 when goals are single atoms, meaning that any such instances can be solved optimally (shortest plans) by running IW $(w)$ in low polynomial time. For example, the goal on $(x, y)$ for any two blocks $x$ and $y$ in Blocks world can be shown to have width no greater than 2 , no matter the number of blocks or initial configuration. This means that IW(2) finds a shortest plan to achieve on $(x, y)$ in polynomial time in the number of atoms and blocks even though the state space for the problem is exponential. The majority of the benchmarks, however, do not feature a single atomic goal but a conjunction of them, and a number of extensions of IW $(k)$ have been developed aimed at them, some of which represent the current state of the art [Lipovetzky and Geffner, 2017]. Moreover, unlike all the classical planning algorithms developed so far, including those that rely on heuristics obtained from relaxations or reductions into SAT, some of these extensions scale up equally well without taking into account the structure of actions (preconditions and effects), meaning that they can plan with simulators [Francès et al., 2017]. In particular, IW(1) has been used effectively as an on-line planner in the Atari video games in two modes: with the memory states of the emulator [Lipovetzky et al., 2015] and with the screen states [Bandres et al., 2018]. In the first case, the features $f_{i}$ are associated with each of the 256 values of the 128 memory bytes; in the second case, the features are the B-PROST pixel features [Liang et al., 2016] defined and motivated by the neural net architecture underlying DQN [Mnih et al., 2015].

\section{Learners and Solvers: Contrasts}

The IW(1) planner and the DQN learner perform comparably well in the Atari games while working in ways that illustrate key differences between learners and solvers: DQN requires lots of training data and time, and then plays very fast, reactively; IW(1) plays out of the box with no training but needs to think a bit before each move. ${ }^{2}$ This is a general characteristic: learners require training, which is often slow, but then are fast; solvers can deal with new problems with no training but after some deliberation. Solvers are thus general, domain independent as they are called, in a way that learners can't be: solvers can deal with new problems from scratch, provided a suitable representation of the problems; learners need experience on related problems.

The differences between model-free learners and modelbased solvers are reminiscent of current accounts in psychology that describe the human mind as made of two interacting systems or processes: a System 1 associated with the "intuitive mind", and a System 2 associated with the "analytical mind" [Evans and Stanovich, 2013]. Kahneman [2011] refers to the two processes as fast and slow thinking. Common characteristics associated with these systems are:

$\begin{array}{cc}\text { System 1 } & \text { System 2 } \\ \text { fast } & \text { slow } \\ \text { associative } & \text { deliberative } \\ \text { unconscious } & \text { conscious } \\ \text { effortless } & \text { effortful } \\ \text { parallel } & \text { serial } \\ \text { automatic } & \text { controlled } \\ \text { heuristic } & \text { systematic } \\ \text { specialized } & \text { general } \\ \text {... } & \ldots\end{array}$

It is hard not to see the parallels between the characteristics associated with Systems 1 and 2 on the one hand, and those of learners and solvers on the other. The processes underlying Systems 1 and 2 in the human mind, however, are not independent: System 2 is assumed to have evolved more recently than System 1 and to be based on it. For activities like solving a Sudoku, writing a proof, or understanding a long text, it is assumed that the analytical System 2 is in command, integrating the suggestions of System 1 that are triggered by

${ }^{2} 0.5$ seconds per move in the rollout version of IW(1). 
clues issued by System 2 or picked up from context. Our account of classical planning as heuristic search has this flavor: the deliberate search for the goal is informed by an heuristic which must be opaque to the "analytical mind", as it is computed from a relaxed model that has no resemblance to the real world [Geffner, 2010]. More generally, there cannot be a System 2 without a System 1, as reasoning is computationally hard and for inference methods to be effective they have to be "biased" to exploit the structure of common tasks. At the same time, human cognition cannot be a System 1 process only, as there are situations and new problems where accumulated experience and built-in mechanisms are not adequate.

\section{Learners and Solvers: Challenges}

The learning algorithm AlphaZero at the heart of the systems that learn to play chess and Go at world-class level by pure self-play [Silver et al., 2017a; 2017b] is an effective integration of a learner and a solver. AlphaZero is a modified, pointwise version of policy iteration, a basic planning algorithm for MDPs and adversarial games where a policy is evaluated and improved iteratively [Bertsekas, 2018]. In AlphaZero, a neural network is used to represent the value and policy functions, and in each iteration both functions are improved incrementally at one state (and at others by generalization) by carrying out a Monte Carlo tree search [Coulom, 2006; Kocsis and Szepesvári, 2006] from such state guided by the current value and policy functions. Interestingly, AlphaZero is similar to an algorithm developed independently at the same time, Expert Iteration (ExIt), that is explicitly cast as an integration of System 1 and System 2 inference [Anthony et al., 2017]. Both AlphaZero and ExIt can also be understood as systems that learn by imitation but with a planner as teacher, and with the ability to iteratively improve the teacher.

Two key questions are what are the inherent limitations of these algorithms, and what else would be needed in order to get a more general integration of System 1 and System 2 inference in AI systems. As mentioned before, a key restriction of learners relying on neural networks is that the size of their inputs $x$ is fixed. This implies that learners cannot emulate solvers even over specific domains. That is, deep learners cannot emulate a classical planner or a domain-specific Blocks world planner unless arbitrary instances can be expressed in finite size. Attention mechanisms have been proposed for this but attention may be more relevant for executing policies than for computing them. Regarding the second question, some key dimensions for a more general and synergistic integration of learners and solvers are:

Model learning. Solvers provide flexibility and generality, but solvers need models. Explanation and accountability also require models: we build explanations and make predictions using models [Darwiche, 2017; Lake et al., 2017; Pearl, 2018]. Model-based reinforcement learning is modellearning but the standard algorithms assume that the state variables are given. Learning models from streams of actions and partial observations remains challenging.

Learning the relevant variables. In deep learning, representation learning is learning functions (features) of the in- puts that are somehow reusable. Features that are particularly reusable are the variables of a problem. For example, many of the Atari games involve 2D objects that move, and things that happen as a result of collisions. How to learn such variables from the screen? Should the moving objects emerge from a general learning scheme, from prior knowledge, or from a combination of both [Mugan and Kuipers, 2012; Thomas et al., 2017; Marcus, 2018a]?

Learning finite-size abstract representations. The input size restriction makes it challenging for DRL methods to learn general policies for achieving a goal like on $(A, B)$ over all instances with blocks $A$ and $B$. An alternative to the use of attention mechanisms is to learn problem abstractions of bounded size that depend on the goal. For example, for achieving the goal on $(x, y)$ the only features that matter are the number of blocks above $x$ and above $y$, along with some booleans, and a convenient, fixed-size abstraction of this family of problems can be obtained by projecting the actions over such features [Bonet and Geffner, 2018]. The challenge is to learn such features and projections automatically.

Most work in DRL has not been aimed at an integration of learners and solvers but at expanding the range of problems handled by DRL. Something similar can be said about work in planners and solvers. Yet, the solutions to some of the bottlenecks faced by each approach, that often have to do with representational issues, may lie in the integration that is required to resemble the integration of Systems 1 and 2 in the human mind. One area where research on solvers and learners meet, as mentioned above, is in the computation of generalized plans capable of solving many problems at the same time. Other types of challenges are dealing with other agents, virtual or human, in what constitutes the social dimension of intelligence, and dealing with a physical and social world that is not segmented into problems or tasks [Dietterich, 2018].

\section{AI: Dreams and Nightmares}

AI is not close to a general, human level-intelligence but this does not mean that current and future AI capabilities can't be used for good or ill. The Asilomar AI Principles enumerate ways in which harms can be avoided and benefits realized $^{3}$ but the principles are not easy to enforce. It would be great to align the values of AI systems with human values, but the same applies to technology in general, politics, and economics. Clearly, there are other forces at play: forces like a powerful market and a weak political system, both increasingly aimed at our System 1. I have argued why flexible and transparent AI systems need a System 2 that cannot be provided by learners alone. The same applies to our collective lives where learning from mistakes, when it is possible at all, can be very costly. For living together in modern societies, we need a well-functioning System 2 informed by facts and the common good. ${ }^{4}$ If we want good AI, we can't look away from culture and politics.

\footnotetext{
${ }^{3}$ https://futureoflife.org/ai-principles.

${ }^{4}$ [Stanovich, 2005; Heath, 2014].
} 


\section{Acknowledgments}

I thank Jérôme Lang for the invitation to speak at IJCAI, Guillem Francès and Blai Bonet for many useful comments, and my current and former students for the opportunity to learn with them. The work is partially funded by grant TIN2015-67959-P, MINECO, Spain.

\section{References}

[Anthony et al., 2017] T. Anthony, Z. Tian, and D. Barber. Thinking fast and slow with deep learning and tree search. In NIPS, 2017.

[Bandres et al., 2018] W. Bandres, B. Bonet, and H. Geffner. Planning with pixels in (almost) real time. In AAAI, 2018.

[Bertsekas, 1995] D. Bertsekas. Dynamic Programming and Optimal Control, Vols 1 and 2. Athena Scientific, 1995.

[Bertsekas, 2018] D. Bertsekas. Feature-based aggregation and deep reinforcement learning: A survey and some new implementations. arXiv preprint arXiv:1804.04577, 2018.

[Biere et al., 2009] A. Biere, M. Heule, H. van Maaren, and T. Walsh, editors. Handbook of Satisfiability: Volume 185 Frontiers in Artificial Intelligence and Applications. IOS Press, 2009.

[Bonet and Geffner, 2001] B. Bonet and H. Geffner. Planning as heuristic search. Artificial Intelligence, 129(12):5-33, 2001.

[Bonet and Geffner, 2014] B. Bonet and H. Geffner. Flexible and scalable partially observable planning with linear translations. In Proc. AAAI, 2014.

[Bonet and Geffner, 2018] B. Bonet and H. Geffner. Features, projections, and representation change for generalized planning. arXiv preprint arXiv:1801.10055, 2018.

[Bonet et al., 2009] B. Bonet, H. Palacios, and H. Geffner. Automatic derivation of memoryless policies and finitestate controllers using classical planners. In Proc. ICAPS09, pages 34-41, 2009.

[Bonet et al., 2017] B. Bonet, G. De Giacomo, H. Geffner, and S. Rubin. Generalized planning: non-deterministic abstractions and trajectory constraints. In Proc. IJCAI, 2017.

[Camacho et al., 2017] A. Camacho, E. Triantafillou, C. Muise, J. Baier, and S. McIlraith. Non-deterministic planning with temporally extended goals: LTL over finite and infinite traces. In Proc. AAAI, 2017.

[Charniak et al., 1980] E. Charniak, C. Riesbeck, and D. McDermott. Artificial intelligence programming. L. Erlbaum Associates, 1980.

[Chatterjee et al., 2016] K. Chatterjee, M. Chmelik, and J. Davies. A symbolic SAT-based algorithm for almostsure reachability with small strategies in POMDPs. In Proc AAAI, 2016.

[Coulom, 2006] R. Coulom. Efficient selectivity and backup operators in Monte-Carlo tree search. In Proc. Int. Conference on Computers and Games, pages 72-83. Springer, 2006.
[Darwiche, 2017] A Darwiche. Human-level intelligence or animal-like abilities? arXiv preprint, 2017.

[Dechter, 2003] R. Dechter. Constraint Processing. Morgan Kaufmann, 2003.

[Dietterich, 2018] T. Dietterich. Reflections on innateness in machine learning. https://medium.com/@tdietterich, 2018.

[Evans and Stanovich, 2013] J. Evans and K. Stanovich. Dual-process theories of higher cognition: Advancing the debate. Perspectives on psychological science, 8(3), 2013.

[Feigenbaum and Feldman, 1963] E.A. Feigenbaum and J. Feldman. Computers and thought. McGraw-Hill, 1963.

[Francès et al., 2017] G. Francès, M. Ramírez, N. Lipovetzky, and H. Geffner. Purely declarative action representations are overrated: Classical planning with simulators. In Proc. IJCAI, 2017.

[Gebser et al., 2012] M. Gebser, R. Kaminski, B. Kaufmann, and T. Schaub. Answer set solving in practice. Morgan \& Claypool, 2012.

[Geffner and Bonet, 2013] H. Geffner and B. Bonet. A concise introduction to models and methods for automated planning. Morgan \& Claypool, 2013.

[Geffner, 2010] H. Geffner. Heuristics, planning and cognition. In J. Halpern R. Dechter, H. Geffner, editor, Heuristics, Probability and Causality. A Tribute to Judea Pearl. College Publications, 2010.

[Genesereth et al., 2005] M. Genesereth, N. Love, and B. Pell. General game playing: Overview of the AAAI competition. AI magazine, 26(2):62, 2005.

[Graves et al., 2013] A. Graves, A. Mohamed, and G. Hinton. Speech recognition with deep recurrent neural networks. In Proc. ICASSP, pages 6645-6649, 2013.

[Graves et al., 2014] A. Graves, G. Wayne, and I. Danihelka. Neural Turing machines. arXiv preprint arXiv:1410.5401, 2014.

[Groshev et al., 2017] E. Groshev, A. Tamar, S. Srivastava, and P. Abbeel. Learning generalized reactive policies using deep neural networks. arXiv preprint arXiv:1708.07280, 2017.

[Heath, 2014] J. Heath. Enlightenment 2.0. Harper Collins, 2014.

[Hoffmann and Nebel, 2001] J. Hoffmann and B. Nebel. The FF planning system: Fast plan generation through heuristic search. JAIR, 14:253-302, 2001.

[Hu and De Giacomo, 2011] Y. Hu and G. De Giacomo. Generalized planning: Synthesizing plans that work for multiple environments. In Proc. IJCAI, 2011.

[Kaelbling et al., 1998] L. Kaelbling, M. Littman, and T. Cassandra. Planning and acting in partially observable stochastic domains. AI, 101(1-2):99-134, 1998.

[Kahneman, 2011] D. Kahneman. Thinking, fast and slow. Farrar, Straus and Giroux, 2011. 
[Kautz and Selman, 1996] H. Kautz and B. Selman. Pushing the envelope: Planning, propositional logic, and stochastic search. In Proc. AAAI, pages 1194-1201, 1996.

[Kaye, 2000] R. Kaye. Minesweeper is NP-Complete. Mathematical Intelligencer, 22(2):9-15, 2000.

[Kocsis and Szepesvári, 2006] L. Kocsis and C. Szepesvári. Bandit based Monte-Carlo planning. In Proc. ECML2006, pages 282-293, 2006.

[Krizhevsky et al., 2012] A. Krizhevsky, I. Sutskever, and G. Hinton. Imagenet classification with deep convolutional neural networks. In NIPS, 2012.

[Lake et al., 2017] B. Lake, T. Ullman, J. Tenenbaum, and S. Gershman. Building machines that learn and think like people. Behavioral and Brain Sciences, 40, 2017.

[LeCun et al., 1989] Y. LeCun, B. Boser, J. Denker, D. Henderson, R. Howard, W. Hubbard, and L. Jackel. Backpropagation applied to handwritten zip code recognition. Neural computation, 1(4):541-551, 1989.

[LeCun et al., 2015] Y. LeCun, Y. Bengio, and G. Hinton. Deep learning. Nature, 521(7553):436, 2015.

[Lenat et al., 1990] D. Lenat, R. Guha, K. Pittman, D. Pratt, and M. Shepherd. CYC: toward programs with common sense. Communications of the ACM, 33(8):30-49, 1990.

[Liang et al., 2016] Y. Liang, M. Machado, E. Talvitie, and M. Bowling. State of the art control of Atari games using shallow reinforcement learning. In Proc. AAMAS, 2016.

[Lipovetzky and Geffner, 2012] N. Lipovetzky and H. Geffner. Width and serialization of classical planning problems. In Proc. ECAI, 2012.

[Lipovetzky and Geffner, 2017] N. Lipovetzky and H. Geffner. Best-first width search: Exploration and exploitation in classical planning. In Proc. AAAI, 2017.

[Lipovetzky et al., 2015] N. Lipovetzky, M. Ramirez, and H. Geffner. Classical planning with simulators: Results on the atari video games. In Proc. IJCAI, 2015.

[Marcus, 2018a] G. Marcus. Innateness, AlphaZero, and artificial intelligence. arXiv preprint, 2018.

[Marcus, 2018b] Gary Marcus. Deep learning: A critical appraisal. arXiv preprint arXiv:1801.00631, 2018.

[McCarthy, 1958] J. McCarthy. Programs with common sense. In Proc. Symp. on Mechanization of Thought Processes, 1958.

[McCarthy, 1987] J. McCarthy. Generality in artificial intelligence. Communications of the ACM, 30(12), 1987.

[McDermott, 1999] D. McDermott. Using regression-match graphs to control search in planning. Artificial Intelligence, 109(1-2):111-159, 1999.

[Mnih et al., 2015] V. Mnih, K. Kavukcuoglu, D. Silver, A. A Rusu, J. Veness, M. G Bellemare, A. Graves, M. Riedmiller, A. K Fidjeland, G. Ostrovski, et al. Humanlevel control through deep reinforcement learning. Nature, 518(7540):529, 2015.
[Mugan and Kuipers, 2012] J. Mugan and B. Kuipers. Autonomous learning of high-level states and actions in continuous environments. IEEE Transactions on Autonomous Mental Development, 4(1):70-86, 2012.

[Norvig, 1992] P. Norvig. Paradigms of artificial intelligence programming: case studies in Common LISP. Morgan Kaufmann, 1992.

[Palacios and Geffner, 2009] H. Palacios and H. Geffner. Compiling uncertainty away in conformant planning problems with bounded width. JAIR, 35:623-675, 2009.

[Pearl, 1983] J. Pearl. Heuristics. Addison Wesley, 1983.

[Pearl, 1988] J. Pearl. Probabilistic Reasoning in Intelligent Systems. Morgan Kaufmann, 1988.

[Pearl, 2018] J. Pearl. Theoretical impediments to machine learning with seven sparks from the causal revolution. arXiv preprint arXiv: 1801.04016, 2018.

[Ramírez and Geffner, 2010] M. Ramírez and H. Geffner. Probabilistic plan recognition using off-the-shelf classical planners. In Proc. AAAI, 2010.

[Rintanen, 2004] J. Rintanen. Complexity of planning with partial observability. In ICAPS, pages 345-354, 2004.

[Rumelhart and McClelland, 1986] D. Rumelhart and J. McClelland, editors. Parallel distributed processing: explorations in the microstructure of cognition. Vol. 1. MIT Press, 1986.

[Russell and Norvig, 2009] S. Russell and P. Norvig. Artificial Intelligence: A Modern Approach. Prentice Hall, 2009. 3rd Edition.

[Silver et al., 2017a] D. Silver, T. Hubert, J. Schrittwieser, I. Antonoglou, M. Lai, A. Guez, M. Lanctot, L. Sifre, D. Kumaran, T. Graepel, et al. Mastering chess and shogi by self-play with a general reinforcement learning algorithm. arXiv preprint arXiv:1712.01815, 2017.

[Silver et al., 2017b] D. Silver, J. Schrittwieser, K. Simonyan, I. Antonoglou, A. Huang, A. Guez, T. Hubert, L. Baker, M. Lai, A. Bolton, et al. Mastering the game of go without human knowledge. Nature, 550(7676):354, 2017.

[Srivastava et al., 2011] S. Srivastava, N. Immerman, and S. Zilberstein. A new representation and associated algorithms for generalized planning. AI, 175(2):615-647, 2011.

[Stanovich, 2005] K. Stanovich. The robot's rebellion: Finding meaning in the age of Darwin. Univ. of Chicago Press, 2005.

[Sutton and Barto, 1998] R. Sutton and A. Barto. Introduction to Reinforcement Learning. MIT Press, 1998.

[Thomas et al., 2017] V. Thomas, J. Pondard, E. Bengio, M. Sarfati, P. Beaudoin, M. Meurs, J. Pineau, D. Precup, and Y. Bengio. Independently controllable features. arXiv preprint, 2017.

[Turing, 1936] A. Turing. On computable numbers, with an application to the Entscheidungsproblem. In Proc. of the London Mathematical Society, volume 42, 1936. 